\title{
PÓS PARTO DE VACAS LEITEIRAS ORIUNDAS DE REBANHOS DA AGRICULTURA FAMILIAR DO MUNICÍPIO DE REALEZA - PARANÁ
}

\footnotetext{
Marina Gabriela Possa ${ }^{1}$, Adalgiza Pinto Neto ${ }^{2}$, Fabricio Bernardi ${ }^{1}$, Marcelo Falci Mota $^{2}$, Antônio Campanha Martinez ${ }^{3}$

${ }^{1}$ Graduandos de Medicina Veterinária. Universidade Federal da Fronteira Sul. Campus Realeza. Realeza-PR.

${ }^{2}$ Docentes do Curso de Medicina Veterinária. Universidade Federal da Fronteira Sul.

Campus Realeza. Realeza-PR. Contato: adalgiza.neto@uffs.edu.br; adalgiza.uffs@gmail.com

${ }^{3}$ Docente da Universidade Estadual de Maringá. Campus Umuarama. UmuaramaPR.

Recebido em: 08/09/2015 - Aprovado em: 14/11/2015 - Publicado em: 01/12/2015 DOI: http://dx.doi.org/10.18677/Enciclopedia_Biosfera_2015_210
}

Objetivou-se avaliar o pós parto de fêmeas bovinas em três propriedades leiteiras da agricultura familiar do Realeza - Paraná, de agosto de 2013 a agosto de 2014. Coletou-se informações zootécnicas dos animais e rebanho, avaliado o ECC dos animais no periparto, parto e pós-parto, onde foi realizada avaliação ginecológica aos 30 e 60 dias pós-parto. Realizou-se análise estatística descritiva de frequência e correlações de referência cruzada. Das vacas, 58,2\% (60/103) apresentaram involução uterina completa até os 60 dias pós-parto e 41,75\% (43/103) não concluíram este evento até esse período. Em 25,24\% (26/103) dos animais foi detectada a presença de corpo lúteo, indicando retorno à ciclicidade, e em $74,75 \%$ (77/103) o corpo lúteo não foi detectado até os 60 dias pós-parto. As correlações indicaram que vacas jovens (de até três anos e de três a cinco anos) apresentaram involução uterina mais precoce que aquelas com mais de cinco anos. Primíparas e vacas de dois a cinco partos apresentaram involução uterina mais precoce que vacas acima de cinco partos. Correlação positiva foi estabelecida entre o ECC dos animais com a presença de corpo lúteo, onde animais com ECC adequado (de 2,5 a $3,5)$ aos 30 dias pós-parto apresentaram maior chance de apresentarem corpo lúteo do que animais com ECC menor que 2,5. A idade e a ordem de partos demonstraram exercer influência no período de involução uterina e o ECC dos animais aos 30 dias pós-parto influenciou no período para a presença de corpo lúteo ovariano.

PALAVRAS-CHAVE: eficiência reprodutiva, involução uterina, presença de corpo lúteo.

\section{PERIOD PUERPERAL OF DAIRY COWS ORIGINATING FROM HERDS OF THE FAMILY FARM IN THE CITY OF REALEZA - PARANÁ}

\author{
ABSTRACT \\ This study objective to evaluate the puerperal period of 103 animals of three dairy \\ farms the family farm in the city of Realeza - Paraná, from august 2013 to august
}


2014. Initially zootechnical informations of animal and herd were collected, measured the animal BCS in peripartum, parturition and postpartum (30 and 60), and performed gynecological evaluation at 30 and 60 days postpartum. Data were subjected to descriptive statistics and correlations of frequency of cross-reference. $58.2 \%$ $(60 / 103)$ of the cows had complete uterine involution up to 60 days postpartum and $41.75 \%$ (43/103) did not complete this event until 60 days postpartum. In $25.24 \%$ $(26 / 103)$ of the animals was detected the presence of corpus luteum, indicating return to cyclicity, and in $74.75 \%(77 / 103)$ the corpus luteum was not detected until 60 days postpartum. The correlations observed in the study indicated that young cows (up to three years and three to five years) had earlier uterine involution than those older (over five years). Primiparous cows and two to five parturitions received earlier uterine involution that cows over five parturition. Positive correlation was established between the body condition score (BCS) of the animals with the presence of corpus luteum, where animals with appropriate ECC at 30 days postpartum were more corpus luteum than lean animals. The age and the order of births demonstrated influence in the period of uterine involution and the ECC of the animals at 30 days post-partum period to influence the presence of corpus luteum.

KEYWORDS: Presence of corpus luteum, uterine involution, reproductive efficiency.

\section{INTRODUÇÃO}

A eficiência reprodutiva dos rebanhos leiteiros, particularmente aqueles inseridos em pequenas propriedades, é um dos pontos críticos para a manutenção dos produtores na atividade (SARTORI, 2007, EMERICK et al., 2010, VIEIRA, 2014). O Brasil, de modo geral, passou por uma evolução tecnológica no setor pecuário, contudo, muitos entraves ainda persistem e são constatados pelos baixos índices reprodutivos (VIEIRA, 2014). A eficiente reprodução do rebanho interfere diretamente na produtividade, e como consequência no retorno econômico da atividade leiteira. Esta eficiência determina maior longevidade dos animais, produtividade (produção de leite e número de bezerros) e progresso genético do rebanho (LEITE et al., 2001, SARTORI, 2007, SAKAGUCHI, 2011, VERGARA et al., 2014). Todavia, o manejo ineficiente reduz os índices reprodutivos, incorrendo em custos para a concepção das vacas e manutenção das mesmas em período seco (MARTINS et al., 2013).

Após o parto, várias mudanças fisiológicas ocorrem no útero das vacas. Para que a fêmea retorne a atividade reprodutiva é necessário um período de recuperação, que pode ser prolongado por inúmeros fatores, tais como idade e ordem de partos que interferem no funcionamento do útero, e, portanto, no desempenho reprodutivo como um todo (ONYANGO, 2014). A fertilidade da vaca no período puerperal depende da involução do útero e do restabelecimento da atividade ovariana por meio do funcionamento do eixo hipotalâmico-hipofisário-ovarianouterino (BUGNER \& ALENCAR 1985, OPSOMER et al., 1996).

O puerpério compreende um período que vai do parto até o aparecimento de estro fértil, passível de iniciar uma nova gestação (HORTA, 1995). Este período é fisiológico, e corresponde a recuperação das alterações nos órgãos reprodutivos da fêmea que ocorrem em decorrência da gestação e parto (KOZICKI, 1998, EMERICK et al., 2010).

O puerpério é um período crítico para a eficiência reprodutiva de uma vaca, e deve evoluir para que a atividade reprodutiva da fêmea esteja normalizada antes dos 60 dias pós-parto (HORTA, 1995, OPSOMER et al., 1996). O acompanhamento e controle do puerpério são imprescindíveis para se atingir uma completa eficiência 
produtiva (MARTINS et al., 2013, SCULLY et al., 2013). Isto permite a identificação precoce dos fatores que causam prejuízos, como por exemplo, animais acíclicos, para que, respeitando a fisiologia dos mesmos, se tenha o menor período de serviço possível e o intervalo de parto próximo a 12 meses (HORTA, 1995, OPSOMER et al., 1996, LEROY et al., 2009). Além disso, vacas com complicações puerperais exigem maior tempo para a involução uterina e retorno à ciclicidade, comprometendo assim os índices produtivos (MARTINS et al., 2013).

Diante do exposto, objetivou-se com este estudo avaliar o período de pós parto de vacas oriundas de rebanhos leiteiros de agricultura familiar do Município de Realeza, Estado do Paraná.

\section{MATERIAL E MÉTODOS}

O estudo foi desenvolvido em três propriedades de agricultura familiar, produtoras de leite do Município de Realeza - Paraná, contando com a avaliação de 103 animais em lactação, das raças Jersey e Holandês, no período de agosto/2013 a agosto/2014. O município de Realeza faz parte da Mesorregião Sudoeste Paranaense com clima subtropical úmido e temperatura média de $19^{\circ} \mathrm{C}$. Se localiza à 520 metros de altitude média, latitude sul $25^{\circ} 46^{\prime} 49^{\prime \prime}$ e longitude oeste $53^{0} 32^{\prime} 37^{\prime \prime}$.

Após a anuência dos proprietários para a realização desse estudo, foram colhidas informações zootécnicas dos animais e do rebanho, como raça, idade, ordem de parto, data do parto, data do primeiro estro pós-parto, período seco, produção média de leite, manejo nutricional e ocorrência de enfermidades no período do estudo (retenção de placenta, deslocamento de abomaso, hipocalcemia, mastite, cetose e anaplasmose/ babesiose).

Todos os animais estudados foram mantidos em pastagem com água ad libitum e receberam suplementação com silagem de milho, concentrado e sal mineral. Todos foram submetidos ao período seco de 60 dias com manejo alimentar pré-parto semelhante, acrescido de suplementação mineral comercial destinada à este período, iniciada aos 20 dias pré parto.

Realizou-se a mensuração do escore de condição corporal (ECC) dos animais no periparto, parto e pós-parto (30 e 60 dias), por meio de visualização, inspeção e palpação da condição corporal, analisando a cobertura muscular e adiposa dos processos transversos das vértebras, da região do íleo, ísquio e inserção da cauda (CORASSIN, 2004). A escala de ECC utilizada variou de um a cinco, aceitando variações de meio ponto. Os animais foram classificados como magros (ECC até 2,5 pontos), adequados $(2,5<\mathrm{ECC}<3,5)$ ou gordos $(\mathrm{ECC}>3,5)$.

Aos 30 e 60 dias pós-parto, realizou-se avaliação transretal e exame de inspeção vaginal com auxilio de espéculo vaginal, onde se considerou: posição uterina (abdominal ou pélvico), tamanho uterino (pequeno, médio ou grande), simetria dos cornos uterinos (simétricos ou assimétricos), presença de conteúdo no útero (cristalino, catarral ou purulento), consistência uterina (flácido ou túrgido), estruturas ovarianas (folículos e/ou corpos lúteos) e presença de corpo lúteo. Considerou-se como involução uterina completa útero simétrico, pequeno, na cavidade pélvica e sem conteúdo.

Os dados coletados foram tabulados e submetidos à análise estatística descritiva, a fim de apresentar os fatores envolvidos com período puerperal das fêmeas bovinas estudadas. Os valores médios e as correlações foram testadas pelo 
programa Estatístico IBM SPSS ${ }^{\circledR} 20.0^{41}$, por meio de análise descritiva de frequência, correlações de tabela de referência cruzada e cálculo de intensidade de associação por meio das Odds Ratio (OR), considerando $5 \%$ de significância $(\mathrm{P}<0,05)$. Para os cálculos de correlação foi aplicado o Teste do Qui-quadrado, corrigido pelo Teste de Fischer.

\section{RESULTADOS}

No periparto, 67\% (69/103) e 33\% (34/103) das fêmeas apresentaram ECC adequado ou gordo, respectivamente, não encontrando correlação entre o ECC dos animais estudados e presença de corpo lúteo $(P=0,465)$. Nesse período, o $E C C$ não se correlacionou a ocorrência concomitante de afecções puerperais $(P=1,00)$. Ao parto, observou-se $11,6 \%$ (12/103), 86,4\% (89/103) e 1,9\% (2/103) dos animais magros, adequados e gordos, respectivamente, sendo que ECC ao parto não exerceu influência sobre a presença de corpo lúteo $(P=0,56)$.

$\mathrm{Na}$ avaliação de ECC aos 30 dias pós-parto foram encontrados animais magros, adequados e gordos, sendo 56,3\% (58/103), 41,7\% (43/103) e 1,9\% (2/103), respectivamente. Ao correlacionar os ECCs dos animais estudados a presença de corpo lúteo, observou-se que animais de ECC adequado apresentaram 0,26 vezes mais chances de apresentar corpo lúteo até 30 dias pós-parto que animais magros $(P=0,006)$, enquanto que a presença de corpo lúteo nos animais de ECC magro comparado ao gordo, e adequado ao gordo, não apresentou diferença significativa ( $P=0,472$ e $P=0,268$, respectivamente).

Aos 60 dias pós-parto, 63,1\% (65/103) dos animais apresentaram-se magros e 36,9\% (38/103) adequados, sendo que a presença de corpo lúteo não se correlacionou ao ECC de animais magros e adequados nesse período $(p=0,096)$. Ao se estimar a perda de ECC no pós-parto, observou-se que dos animais que apresentaram perda de ECC acima de um ponto no período pós-parto, $66 \%(68 / 103)$ não apresentaram corpo lúteo, em relação àqueles animais que perderam menos de um ponto no ECC $(P=0,337)$.

A posição, tamanho, simetria, presença de conteúdo e consistência uterina aos 30 e 60 dias pós-parto encontram-se demonstrados na Figura 1, revelando que $75,7 \%(78 / 103)$ e $30,1 \%$ (31/103) dos animais estavam com o útero abdominal e, $24,3 \%(25 / 103)$ e $69,9 \%$ (72/103) pélvicos, respectivamente. O tamanho do útero aos 30 e 60 dias pós-parto foi distribuído em 25,2\% (26/103) e 68\% (70/103) pequenos, 68,9\% (71/103) e 32\% (33/103) médios e 5,8\% (6/103) e $0 \%$ grandes, respectivamente. Aos 30 dias pós-parto 6,8\% (7/103) das vacas apresentaram simetria uterina, enquanto que aos 60 dias 53,4\% (55/103) delas apresentaram os cornos uterinos simétricos.

O conteúdo uterino observado em 86,4\% (89/103) e 94,2\% (97/103) encontrava-se cristalino, $7,8 \%$ (8/103) e 3,9\% (4/103) purulento, e 5,8\% (6/103) e $1,9 \%$ (2/103) catarral, aos 30 e 60 dias pós-parto, respectivamente (Figura 1). A consistência do útero revelou que 17,5\% (18/103) e 5,8\% (6/103) das vacas estavam com útero flácido e, 82,5\% (95/103) e 94,2\% (97/103) túrgido, aos 30 e 60 dias pósparto respectivamente (Figura 1).

\footnotetext{
${ }^{4} \mathrm{IBM}^{\circledR}$ Corp. Released. SPSS ${ }^{\circledR}$ Statistics for Windows. Version 20.0, Release 20.0.0. Armonk, New York: IBM Corp., 2011.
} 


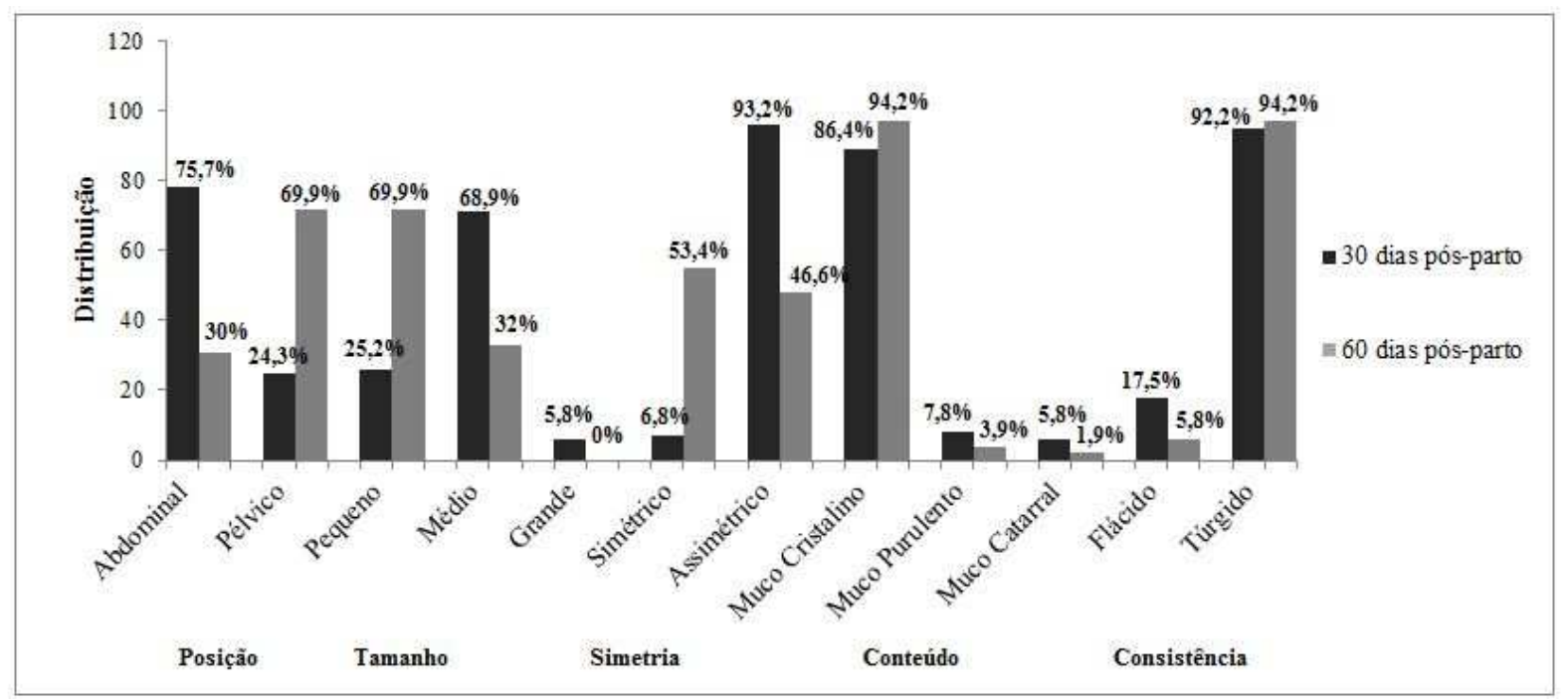

FIGURA 1: Posição, tamanho, simetria, presença de conteúdo e consistência do útero de vacas leiteiras oriundas de rebanho da agricultura familiar, aos 30 e 60 dias pós-parto.

O útero completamente involuído foi observado em 6,8\% (7/103) e 51,4\% (53/103) das vacas aos 30 e 60 dias pós-parto, sendo que $41,8 \%$ (43/103) das vacas não apresentaram involução uterina completa até 60 dias pós-parto. Não foi observada diferença entre as Raças Holandesa (85,5\% - 85/103 animais) e Jersey $(17,5 \%(18 / 103)$ com relação ao período de involução uterina $(P=0,799)$ e a presença de corpo lúteo $(P=0,764)$.

Observou-se que vacas com idade de até três anos apresentaram 3,29 vezes mais chances de apresentarem involução uterina completa até os 60 dias pós-parto do que aquelas com idade superior a cinco anos de idade $(P=0,028)$. Ao se categorizar, vacas com idade entre três a cinco anos apresentaram 3,1 mais chances de apresentarem involução uterina completa até os 60 dias pós-parto que vacas acima de cinco anos de idade $(P=0,032)$.

De maneira semelhante à idade, vacas primíparas apresentaram 6,4 vezes mais chances de terem involução uterina até os 60 dias pós-parto que vacas com mais partos $(P=0,004)$, enquanto que vacas que pariram de duas a cinco vezes apresentaram 3,96 vezes mais chances de terem involução uterina até os 60 dias pós-parto que vacas com mais de cinco partos $(P=0,019)$. No entanto, fêmeas primíparas e vacas de dois a cinco partos apresentaram as mesmas chances de terem involução uterina até os 60 dias pós-parto $(P=0,464)$.

A avaliação ovariana transretal revelou que $71,8 \%(74 / 103)$ e $65 \%(67 / 103)$ das vacas apresentavam crescimento folicular, 3,9\% (4/103) e 21,4\% (22/103) corpo lúteo, e 24,3\% (25/103) e 13,6\% (14/103) nenhuma estrutura ovariana, aos 30 e 60 dias pós-parto, respectivamente. $74,75 \%$ (77/103) dos animais estudados não apresentaram corpo lúteo nos períodos avaliados (Figura 2). 


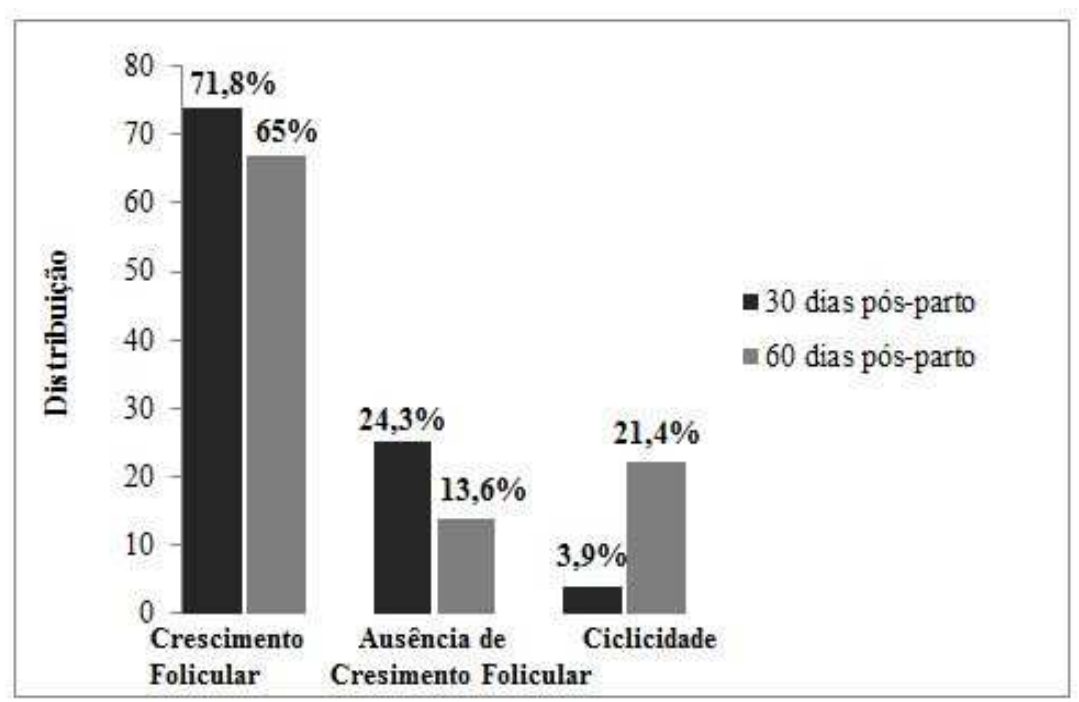

FIGURA 2: Crescimento folicular e presença de corpo lúteo ovariano avaliados através da palpação transretal, em vacas leiteiras oriundas de rebanhos da agricultura familiar, aos 30 e 60 dias pós-parto.

Não se observou correlação da presença de corpo lúteo com a idade (até três anos, três a cinco anos e acima de cinco anos) e com a ordem de partos (primíparas, vacas de dois a cinco partos e vacas acima de cinco partos) $(P>0,05)$. A produção de leite dos rebanhos estudados variou de 25 a 30 litros/vaca/dia e acima de 30 litro/vaca/dia. Não se observou correlação da produção leiteira com a involução uterina e presença de corpo lúteo $(P>0,05)$.

Das enfermidades relatadas no puerpério, 14,6\% (15/103) foram retenção de placenta, $1,94 \%$ (2/103) deslocamento de abomaso, 1,94\% (2/103) mastite, 0,97\% $(1 / 103)$ hipocalcemia, $0,97 \% \quad(1 / 103)$ cetose e $0,97 \%(1 / 103)$ Babesiose/Anaplasmose. A ocorrência dessas enfermidades relatadas não influenciou o período de involução uterina $(p=0,356)$.

Dos 15 animais com retenção de placenta 73,3\% (11/15) apresentaram alteração na secreção cervico-vaginal aos 30 dias pós-parto, sendo $26,6 \%(4 / 15)$ secreção catarral e 46,6\% (7/15) purulenta. Aos 60 dias pós-parto, 13,3\% (2/15) dos animais apresentaram secreção muco purulento, 6,6\% (1/103) muco catarral e $80 \%$ (12/103) muco cristalino.

Observou-se que $0,97 \%$ (1/103) das vacas apresentou período de serviço inferior a 50 dias, 22,3\% (23/103) de 50 a 100 dias, $15,5 \%(16 / 103)$ de 100 a 150 dias, $17,5 \%$ (18/103) de 150 a 200 dias, 8,7\% (9/103) de 200 a 250 e 36\% (37/103) apresentaram período de serviço superior à 250 dias. O período de serviço não se correlacionou ao ECC no periparto, parto e pós-parto (30 e 60 dias) $(P>0,05)$.

\section{DISCUSSÃO}

A ausência de efeito do ECC no periparto e parto sobre a presença de corpo lúteo poderia ser explicada pela adequada condição corporal dos animais estudados, uma vez que acima de $88,35 \%$ (91/103) apresentaram ECC adequado ou gordo, no periparto e parto, e somente $11,6 \%$ (12/103) magros ao parto, corroborando com SAUT et al. (2011) ao relatarem que a boa condição corporal dos 
animais não interfere significativamente com o período puerperal, e com SARTORI (2007) ao descrever que animais mais magros tem menor aporte energético para retornarem a ciclicidade pós-parto.

Aos 30 dias pós-parto verificou-se que animais de ECC adequado apresentaram presença de corpo lúteo superior a observada em animais magros ( $p$ $=0,006)$. Vacas que apresentaram baixo ECC pós-parto tem atraso no retorno da atividade ovariana luteal cíclica, por exigirem mais tempo para o desenvolvimento folicular, devido a necessidade energética (CORASSIN, 2004, SARTORI, 2007, CARVALHO, 2009, BREDA et al., 2014, CARVALHO et al., 2014). A primeira ovulação pós-parto passa de 15-30 dias para 70-100 dias pós-parto ao se comparar animais com boa condição corporal e animais magros (CROWE, 2008, MASLEV et al., 2014). Vacas quando bem nutridas devem demonstrar pelo menos um estro até os 30 dias pós-parto (KOZICKI, 1998, CROWE, 2008, ONYANGO, 2014).

Vacas com condição corporal inferior aos 30 dias pós-parto podem demonstrar crescimento folicular, como observado em $71,8 \%(74 / 103)$ dos animais estudados. No entanto, a presença de corpo lúteo foi verificada em 3,9\% (4/103) dos animais com 30 dias pós-parto, e poderia ser explicada pelo comprometimento da energia necessária para a retomada da liberação de FSH e LH após um período de feed back negativo (SAVIO et al., 1990, BEAM \& BUTLER, 1997, MASLEV et al., 2014), uma vez que $63,1 \%(65 / 103)$ dos animais se encontravam magros. A resposta com liberação de FSH ocorre entre cinco a sete dias pós-parto e para LH entre 25 a 30 dias pós-parto (KOZICKI, 1998). Entre quatro a 10 dias pós-parto ocorre crescimento folicular (SENGER, 2003, MASLEV et al., 2014), porém a ovulação só ocorre quando a disponibilidade energética for suficiente para liberação de picos do hormônio luteinizante (LH) (KOZICKI, 1998, CARVALHO, 2009, SAKAGUCHI, 2011, MASLEV et al., 2014).

A relação entre animais de ECC acima de 2,5, aos 30 dias pós-parto, não interferiu na presença de corpo lúteo, embora observou-se que animais com escore maior que 3,5, mesmo não possuindo condição corporal ideal, apresentariam maior disponibilidade energética para ciclicidade, uma vez que a condição corporal influencia na ciclicidade ovariana (CARVALHO, 2009). No entanto, somente 1,9\% (2/103) dos animais se encontravam gordos aos 30 dias pós-parto, o que poderia ter contribuído para o resultado observado.

O ECC dos animais aos 60 dias pós-parto também não interferiu com a presença de corpo lúteo, sendo que 63,1\% (65/103) deles estavam magros. Nesse período somente $21,4 \%(22 / 103)$ dos animais apresentaram corpo lúteo ovariano que poderia ser explicado pela perda de condição corporal neste período (SARTORI, 2007, BITTAR et al., 2014, CAMPOS et al., 2012, OLIVEIRA et al., 2014).

Até os 60 dias pós-parto, 66\% (68/103) das vacas estudadas perderam pelo menos um ponto no ECC. CARVALHO et al. (2014) relataram que todas ou quase todas as vacas perdem ECC pós-parto, diferindo no grau de perda, devido ao redirecionamento dos nutrientes para a produção de leite (CORASSIN, 2004, LEROY et al., 2009), cuja perda pode ser potencializada pela baixa capacidade de ingestão de alimentos no pós-parto (EMERICK et al., 2010, SANTOS, 2010).

Observou-se ainda que a porcentagem de ECC dos animais estudados, aos 30 e 60 dias pós-parto aumentou com sentido a redução da condição corporal desses animais, uma vez que a porcentagem daqueles animais adequados e gordos foi reduzida, de $1,9 \%$ para $0 \%$ e de $41 \%$ para $36,9 \%$, respectivamente. Em contrapartida, a porcentagem de animais com escore menor que 2,5 aumentou de $56,3 \%$ para $63,1 \%$ aos 30 e 60 dias pós-parto, respectivamente. Esses dados 
poderiam explicar a ausência de corpo lúteo nesses animais, como descrito por CROWE (2008) e MASLEV et al., (2014). Além disto, vacas mais magras apresentam restrição energética, retardando o retorno à ciclicidade (BUGNER \& ALENCAR, 1985).

Os animais estudados que apresentaram perda no ECC acima de um ponto, ou menos de meio ponto até 60 dias pós-parto, não apresentaram diferença na presença de corpo lúteo $(p>0,05)$ que foi superior aos 60 dias pós-parto, independente do grau de perda de ECC, em 78,64\% (81/103) dos animais. Em geral, vacas que ganham ou mantém ECC pós-parto apresentam fertilidade superior (BREDA et al., 2014) que vacas que apresentam perda de condição corporal no puerpério (SARTORI, 2007).

EMERICK et al. (2010) relataram que vacas que perdem até 0,5 unidade de ECC ovulam nos primeiros 30 dias pós-parto, enquanto as que perdem acima de uma unidade ovulam cerca de 50 dias. Embora tenha se observado a presença de corpo lúteo ovariano em 21,4\% (22/103) dos animais estudados, é coerente supor que o intervalo de avaliação ovariana e/ou imprecisão da técnica utilizada poderia ter interferido nesse resultado, uma vez que a avaliação de corpos lúteos inclusos no parênquima ovariano sofre grande margem de erro, pela dificuldade de percepção (NASCIMENTO et al., 2003).

Dos animais estudados, 21,3\% (22/103) apresentaram alguma enfermidade até 60 dias pós-parto, sendo $9 \%(2 / 22)$ de origem metabólica (cetose e hipocalcemia) e as demais relacionadas a fatores como tipo da dieta e manejo sanitário. Observou-se também ausência de correlação entre ECC dos animais no periparto e ocorrência de afecções puerperais, embora CARVALHO (2009) relate que vacas com condição corporal pobre no periparto sofrem com Balanço Energético Negativo no pós-parto. Já ONYANGO (2014) e VERGARA et al. (2014) relataram que o baixo ECC das vacas no periparto leva a imunossupressão, tornando-as mais vulneráveis à problemas pós-parto, aumentando o risco de distocias, distúrbios metabólicos e retenção de placenta. A ausência dessa correlação talvez possa ser explicada pelo ECC adequado (67\% - 69/103) e gordo (33\% - 34/103) dos animais nesse período.

Com o avançar do período pós-parto, observou-se que o tamanho do útero diminuiu, sendo que aos 30 e 60 dias pós-parto, 5,8\% (6/103) e 25,2\% (26/103), e $0 \%$ e $68 \%(70 / 103)$ dos animais apresentaram útero grande e pequeno, respectivamente, contribuindo assim para o processo de involução uterina. A redução do tamanho do útero no puerpério ocorre por contrações miometriais e regeneração tecidual (SENGER, 2003, MARTINS \& BORGES, 2011), sendo que nesse período o útero deve perder peso e diminuir para que a involução uterina se estabeleça (ONYANGO, 2014).

Aos 30 e 60 dias pós-parto, 75,7\% (78/103) e 30,1\% (31/103) das vacas estudadas, respectivamente, apresentavam útero na cavidade abdominal. $A$ permanência do útero na cavidade abdominal deveria ser restrita à primeira semana pós-parto (MARTINS \& BORGES, 2011). No entanto, observou-se inversão da relação entre útero abdominal/pélvico, aos 30 dias e 60 dias pós-parto, indicando atraso no retorno do útero a posição normal não gestante, e consequentemente atraso no processo de involução uterina. BUGNER \& ALENCAR (1985) relataram dados mais animadores ao desse estudo, em que $52,5 \%$ das vacas aos dez dias após o parto apresentavam útero na cavidade abdominal, e aos 25 dias pós-parto todas as 160 vacas estudadas apresentaram útero pélvico. 
A simetria dos cornos uterinos foi observada em 53,4\% (55/103) dos animais aos 60 dias pós-parto, sendo que os 46,6\% (48/103) restantes apresentaram assimetria leve. A simetria entre os cornos uterinos se refere à redução do tamanho do corno uterino em que se estabeleceu a gestação, com redução de volume e perda tecidual, não sendo considerado necessariamente, simetria perfeita (MARTINS \& BORGES, 2011). A medida que aumenta a ordem de partos, ocorre um desgaste do endométrio uterino tornando a involução mais difícil, sendo que o corno uterino que desenvolveu maior número de gestações apresenta menor capacidade de involução que o corno contralateral (BUGNER \& ALENCAR, 1985).

A consistência do útero avaliada aos 30 e 60 dias pós-parto demonstrou que $82,5 \%$ (95/103) e 94,2\% (97/103) das vacas apresentaram útero túrgido, podendo ser decorrente do estímulo estrogênico oriundo do crescimento folicular observado na maioria dos animais. Quanto mais precoce for o crescimento folicular pós-parto, maior será o estímulo capaz de acelerar o processo de involução uterina, devido às contrações uterinas mediadas pelo estrógeno (HORTA, 1995, MARTINS \& BORGES, 2011, MASLEV et al., 2014), além de favorecer a produção de muco e abertura da cérvice para limpeza do útero, com aumento também na defesa imunológica (HORTA , 1995; KOZICKI, 1998).

O conteúdo uterino avaliado demonstrou que aos 30 e 60 dias pós-parto $86 \%$ (89/103) e 94,2\% (97/103) dos animais apresentaram muco cristalino, sendo que a avaliação puerperal precoce do conteúdo uterino com vaginoscópio é um excelente indicativo da contaminação do interior do útero (HORTA, 1995, WILLIAMS, 2013), e por consequência do processo de involução uterina. O útero saudável, entre 7-10 dias pós-parto é capaz de expulsar a maior parte dos agentes infecciosos, e do volume de conteúdo uterino (OPSOMER et al., 1996, KOZICKI, 1998).

SHELDON et al. (2009) e SCULLY et al. (2013) afirmaram que a involução uterina ocorre em média aos 25-50, dias pós-parto. No entanto, neste estudo $41,75 \%$ (43/103) dos animais não completou o processo de involução uterina até os 60 dias pós-parto, onde 5,8\% (6/103) dos animais apresentaram útero de consistência flácida, 5,8\% (6/103) com conteúdo catarral ou purulento, 30\% (31/103) com posição abdominal, 32\% (33/103) com tamanho médio e 46,6\% (50/103) com cornos assimétricos.

A diferenciação entre as Raças Holandesa e Jersey dos animais estudados não se correlacionou com o processo de involução uterina e com a presença de corpo lúteo $(P>0,05)$. Existem especulações controversas sobre o envolvimento racial na involução uterina (KOZICKI, 1998). Relatos indicam que ocorre interferência da aptidão dos animais no processo de involução uterina, onde vacas zebuínas de corte são mais precoces que raças taurinas de aptidão leiteira quanto à involução uterina (BUGNER \& ALENCAR, 1985, HORTA, 1995, MARTINS \& BORGES, 2011).

Outro fator, como a idade da vaca esta relacionado com o processo de involução uterina (BREDA et al., 2014, VIEIRA, 2014). Dado semelhante foi observado nesse estudo, onde vacas com idade superior a cinco anos apresentaram retardo na involução uterina quando comparadas as demais. HORTA (1995) relata que animais mais velhos apresentam fibrose e relaxamento dos ligamentos de sustentação do útero, que dificultam sua regressão e retorno à cavidade pélvica (MARTINS \& BORGES, 2011, MARTINS et al., 2013), uma vez que a involução do útero é atribuída a contrações circulares e longitudinais das fibras musculares lisas do miométrio, que pode sofrer alterações com a idade (BUGNER \& ALENCAR, 1985). ONYANGO (2014) acrescentou que o efeito da idade é ainda mais intenso no 
puerpério, interferindo tanto na involução uterina, quanto no retorno à ciclicidade. No entanto, nesse estudo a idade das fêmeas (5,0 \pm 1,9 anos) não interferiu na presença de corpo lúteo ovariano.

A ordem de partos interferiu na involução uterina das vacas desse estudo, onde fêmeas primíparas apresentaram 6,4 vezes mais chances de terem seus úteros involuídos aos 60 dias pós-parto, que fêmeas que pariram mais vezes. BUGNER \& ALENCAR (1985) e JAINUDEEN \& HAFEZ (2004) tiveram conclusões semelhantes. A cada parto ocorre destruição parcial e gradativa no endométrio uterino, necessitando de mais tempo para completar o processo de involução uterina (BUGNER \& ALENCAR, 1985).

A ordem de partos não é determinante para o retorno à ciclicidade (BUGNER \& ALENCAR, 1985). Pelo contrário, a maturidade fisiológica, atingida com a ordem de partos é importante para determinar o retorno à ciclicidade (PRATA et al., 2014). A ausência de correlação entre a ordem de partos e presença de corpo lúteo também foi confirmada nesse estudo.

A avaliação ovariana indicou que $71,8 \%$ (74/103) e 65\% (67/103) das vacas apresentaram crescimento folicular, 3,9\% (4/103) e 21,4\% (22/103) corpo lúteo e, $24,3 \%$ (25/103) e 13,6\% (14/103) ovários lisos, aos 30 e 60 dias pós-parto, respectivamente. BUGNER \& ALENCAR (1985) encontraram crescimento folicular somente após 20 dias da parição, sendo que aos 40 dias após o parto 37,5\% das vacas apresentaram os ovários sem crescimento folicular perceptível. KOZICKI (1998) relatou que aos 30 dias pós-parto o corpo lúteo deve estar presente em $60 \%$ das vacas que não apresentaram nenhuma anormalidade ovariana. A presença de corpo lúteo ovariano foi observada em 25,3\% (26/103) das vacas estudadas, talvez possa ser explicado pelo intervalo de 30 dias para as avaliações ovarianas, e/ou pela imprecisão da avaliação transretal em se detectar corpos lúteos inclusos (NASCIMENTO et al., 2003).

Não houve correlação entre produção leiteira média e a presença de corpo lúteo. ONYANGO (2014) demonstrou correlação com a ciclicidade, em vacas de maior idade e produção de leite, que apresentaram maior dificuldade de retorno à ciclicidade. É possível que as vacas desse estudo, por apresentaram produção de leite similar, relativamente alta para a região (25 a 30 litros/vaca/dia) não tenham recebido alimentação suficiente para recuperar as perdas verificadas no puerpério, tendo como consequência a redução da atividade hipofisária, levando à redução na função ovariana até a ovulação, como observado em quase 75\% (77/103) dos animais que apresentaram ovários inativos ou com crescimento folicular, corroborando com as descrições de BUGNER \& ALENCAR (1985), KOZICKI (1998) e MASLEV et al., (2014). Acrescenta-se a essa possibilidade, a perda do ECC observada em $66 \%$ (68/103) dos animais estudados até os 60 dias pós-parto.

A inexistência de correlação significativa entre produção de leite das vacas e a involução uterina obtida no presente estudo corrobora com os achados de SCULLY et al. (2013), que demonstraram diferença marcante no metabolismo de vacas lactantes e não-lactantes, sem que a lactação exercesse efeito sobre a involução uterina.

Dos $21,3 \%(22 / 103)$ dos animais que apresentaram alguma enfermidade no puerpério, 68,18\% (15/22) apresentaram retenção de placenta. As enfermidades puerperais são consideradas fatores que interferem na involução uterina (HORTA, 1995, SARTORI, 2007, BREDA et al., 2014). KOZICKI (1998) afirma que anormalidades pós-parto como distocia, retenção de placenta, hipocalcemia, cetose, metrite, cesariana e fetotomia podem prolongar a involução uterina. No entanto, a 
porcentagem de animais desse estudo que apresentou alguma enfermidade no puerpério não influenciou o período de involução uterina.

A retenção de placenta, como verificada em 14,6\% (15/103) das vacas desse estudo, representa um fator de risco para infecção uterina (HORTA, 1995, WILLIAMS, 2013, KRAUSE et al., 2014, ONYANGO, 2014), observada em pelo menos $20 \%$ dos animais que apresentaram alteração na secreção cervico-vaginal aos 60 dias pós-parto. No entanto, é notável a tendência da fêmea em eliminar esta contaminação por conta própria no decorrer do puerpério (SHELDON et al., 2006, SAUT et al., 2011, ONYANGO, 2014), comprovada em $80 \%$ dos animais que apresentaram secreção cristalina ao final desse estudo.

BUGNER \& ALENCAR (1985) relataram que o período de serviço pode sofrer influência do ECC dos animais antes e após o parto, da elevada produção leiteira e do período puerperal fisiológico ou patológico. Entretanto, não foi encontrada correlação da influência destas variáveis no período de serviço apresentado pelos animais desse estudo. Possivelmente, o período de serviço prolongado (superior a 100 dias), observado em 76,69\% (24/103) dos animais desse estudo seja decorrente de aspectos culturais, fortemente ligados ao produtor de leite da Região Sudoeste do Paraná, relativos ao manejo reprodutivo, baseado na detecção de estro e inseminação artificial tardia.

\section{CONCLUSÕES}

Nas condições desse estudo, utilizando-se vacas leiteiras oriundas de rebanhos da agricultura familiar, concluiu-se que: o ECC dos animais no periparto, parto e 60 dias pós-parto não se correlacionou com a presença de corpo lúteo; animais com ECC adequado têm mais chances de ciclar aos 30 dias pós-parto que animais magros ou gordos; a involução uterina completa ocorreu em 58,2\% (60/103) dos animais, sendo que em 6,8\% (7/103) aos 30 dias e em 51,4\% (53/103) ocorreu aos 60 dias pós-parto; animais mais jovens e com menores números de partos apresentam mais chances de completarem a involução uterina; a raça e ocorrência de enfermidades pós-parto não se correlacionaram com o período de involução uterina e/ou presença de corpo lúteo, e o período de serviço não se correlacionou com o ECC dos animais nos períodos estudados.

\section{REFERÊNCIAS}

BEAM, S.W.; BUTLER, W.R. Energy balance and ovarian follicle development prior to the first ovulation postpartum in dairy cows receiving three levels of dietary fat. Biol. Reprod., v.56, p.133-142, 1997.

BITTAR, J.H.J.; PINEDO, P.J.; RISCO, C.A.; SANTOS, J.E.P.; THATCHER, W.W.; HENCKEN, K.E.; CROYLE, S.; GOBIKRUSHANTH, M.; BARBOSA, C.C.; VIEIRANETO, A.; GALVÃO, K.N. Inducing ovulation early postpartum influences uterine health and fertility in dairy cows. J. Dairy Sci., v.97, p.558-3569, 2014.

BREDA, J.C.S.; KOZICKI, L.E.; GREBOGI, A.M.; WEISS, R.R.; BERTOL, M.A.F.; SEGUI, M.S.; PEREZ, J.A.R.; PAGNONCELLI, R. Productive and reproductive parameters in dairy cows and the relationship with ovarian follicular dynamics in the puerperium. Asian J. Agricult. Food Sci., v.2, p.83-88, 2014. 
BUGNER, M.; ALENCAR, M.M. Involução uterina. Pesq. Agrop. Bras., v.20, p.883888, 1985.

CAMPOS, C.C.; RESENDE, E.V.; CARNEIRO, L.C.; MUNHOZ, A.K.; SANTOS, R.M. Condição ovariana de vacas holandesas no início do programa reprodutivo. Vet. Not., v.18, p.145-147, 2012.

CARVALHO, B.C. Parâmetros reprodutivos, metabólitos e produção de leite de vacas mestiças Holandês × Zebu submetidas a dois manejos pré-parto. 2009. 193p. Tese (Doutorado) - Escola de Veterinária - Universidade Federal de Minas Gerais, Belo Horizonte, 2009.

CARVALHO, P.D.; SOUZA, A.H.; AMUNDSON, M.C.; HACKBART, K.S.; FUENZALIDA, M.J.; HERLIHY, M.M.; AYRES, H.; DRESCH, A.R.; VIEIRA, L.M.; GUENTHER, J.N.; GRUMMER, R.R.; FRICKE, P.M.; SHAVER, R.D.; WILTBANK, M.C. Relationships between fertility and postpartum changes in body condition and body weight in lactating dairy cows. J. Dairy Sci., v.97, p.3666-3683, 2014.

CORASSIN, C.A. Determinação e avaliação de fatores que afetam a produtividade de vacas leiteiras: aspectos sanitários e reprodutivos. 2004. 113p. Tese (Doutorado) - Escola Superior de Agricultura "Luiz de Queiroz" Universidade de São Paulo, Piracicaba, 2004.

CROWE, M.A. Resumption of ovarian cyclicity in post-partum beef and dairy cows. Reprod. Domest. Anim., v.43, p.20-28, 2008.

EMERICK, L.L.; DIAS, J.C.; GONÇALVES, P.E.M.; MARTINS, J.A.M.; SOUZA, F.A.; VALE FILHO, V.R.; ANDRADE, V.J. Retorno da atividade ovariana luteal cíclica de vacas de corte no pós-parto: uma revisão. Rev. Bras. Reprod. Anim., v.33, p.203$212,2010$.

HORTA, A.E.M. Fisiologia do puerpério na vaca. In: $8^{\text {a }}$ JORNADAS INTERNACIONALES DE REPRODUCCIÓN ANIMAL. 1995. Anais... Santarém: Portugal, 1995. p.50.

JAINUDEEN, M.R.; HAFEZ, S.E. Bovinos e bubalinos. In: HAFEZ, E.S.E.; HAFEZ, B. (eds.) Reprodução animal. 7.ed. Barueri, São Paulo. 2004. p.159-171

KOZICKI, L. E. Aspectos fisiológicos do puerpério em bovinos. Arch. Vet. Scienc., v.3, p.9-19, 1998.

KRAUSE, A.R.; PFEIFER, L.F.M.; MONTAGNER, P.; WESCHENFELDER, M.M.; SCHWEGLER, E.; LIMA, M.E.; XAVIER, E.G.; BRAUNER, C.C.; SCHMITT, E.; DEL PINO, F.A.B.; MARTINS, C.F.; CORRÊA, M.N.; SCHNEIDER, A. Associations between resumption of postpartum ovarian activity, uterine health and concentrations of metabolites and acute phase proteins during the transition period in Holstein cows. Anim. Reprod. Sci., v.145, p.8-14, 2014.

LEITE, T.E.; MORAES, J.C.F; PIMENTEL, C.A. Eficiência produtiva e reprodutiva em vacas leiteiras. Ciênc. Rural, v.31, p.467-472, 2001. 
LEROY, J.L.M.R.; VANHOLDER, T.; VAN KNEGSEL, A.T.M.; GARCIA-ISPIERTO, A.; BOLS, P.E.J. Priorização de nutrientes em vacas leiteiras no pós-parto imediato: discrepância entre metabolismo e fertilidade. In: XII CURSO NOVOS ENFOQUES NA PRODUÇÃO E REPRODUÇÃO DE BOVINOS, 2009. Anais... Uberlândia, 2009. p.13.

MARTINS, T.M.; BORGES, Á.M. Avaliação uterina em vacas durante o puerpério. Rev. Bras. Reprod. Anim., v.35, p.433-443, 2011.

MARTINS, T.M.; SANTOS, R.L.; PAIXÃO, T.A.; COSTA, É. A.; PIRES, A.C.; BORGES, Á. M. Aspectos produtivos e reprodutivos de vacas da raça holandesa com puerpério normal ou patológico. Arq. Bras. Med. Vet. Zootec., v.65, p.13481356, 2013.

MASLEV, T.S.; HRISTOVA, T.S.; STOYCHEVA, S. Study on the performance of gonadotropin-releasing hormone $(\mathrm{GnRH})$ in the puerperal period of beef cows. Biotechnology in Anim. Husbandry., v.30, p.29-36, 2014.

NASCIMENTO, A.A.; PINHEIRO, N.L.; SALES, A.; VIANA, J.H.M. Correlação morfométrica do ovário de fêmeas bovinas em diferentes estádios reprodutivos. Braz. J. Vet. Res. Anim. Sci., v.40, p.126-132, 2003.

OLIVEIRA, R.S.B.R.; MOURA, A.R.F.; PÁDUA, M.F.S.; BARBON, I.M.; SILVA, M.E.M.; SANTOS, R.M.; MUNDIM, A.V.; SAUT, J.P.E. Perfil metabólico de vacas mestiças leiteiras com baixo escore de condição corporal no periparto. Pesq. Vet. Bras., v.34, p.362-368, 2014.

ONYANGO, J. Cow postpartum uterine infection: A review of risk factors, prevention and the overall impact. Veterinary Research International, v.2, p.18-32, 2014.

OPSOMER, G., MIJTEN, P., CORYN, M. \& KRUIF, A. Post-partum anoestrus in dairy cows: a review. Veterinary Quarterly, v.18, p.68-75, 1996.

PRATA, M.A.; PEREIRA, M.C.; EL FARO, L.; CARDOSO, V.L.; FILHO, A.E.V.; VERNEQUE, R.S.; BRUNELI, F.A.T.; PEIXOTO, M.G.C.D. Efeito do intervalo de partos sobre a eficiência produtiva e econômica em rebanhos Gir Leiteiro. B. Indústr. Anim., v.71, p.1-7, 2014.

SAKAGUCHI, M. Pratical aspects of the fertility of dairy cattle. J. Reprod. Development, v.57, p.17-33, 2011.

SANTOS, E.P. 2010. Uso de gordura em dietas de vacas no pré-parto e em lactação. In: XII CURSO NOVOS ENFOQUES NA PRODUÇÃO E REPRODUÇÃO DE BOVINOS, 2010. Anais... Uberlândia, 2010. p.20.

SARTORI, R. Manejo reprodutivo da fêmea bovina. Reprod. Anim., v.31, p.153-159. 2007. 
SAUT, J.P.E.; OLIVEIRA, R.S.B.R.; MARTINS, C.F.G.; MOURA, A.R.F.; TSURUTA, S.A.; NASCIUTTI, N.R.; SANTOS, R.M.; SELWYN, A.H. Clinical observations of postpartum uterine involution in crossbred dairy cows. Vet. Not., v.17, p.16-25, 2011.

SAVIO, J.D.; BOLAND, M.P.; HYNES, N.; ROCHE, J.F. Resumption of follicular activity in the early post-partum period of dairy cows. J. Reprod. Fert., v.88, p.569$579,1990$.

SCULLY, S.; MAILLO, V.; DUFFY, P.; KELLY, A.K.; CROWE, M.A.; RIZOS, D.; LONERGAN, $P$. The effect of lactation on post-partum uterine involution in Holstein dairy cows. Reprod. Dom. Anim., v.48, p.888-892, 2013.

SENGER, P.L. The puerperium and lactation. In: SENGER, P.L. (ed) Pathways to pregnancy and parturition. Washington: Current Conceptions, 2003, p.326-345.

SHELDON, I.M.; LEWIS, G.S.; LEBLANC, S.; GILBERT, R.O. Defining postpartum uterine disease in cattle. Theriogenology, v.65, p.1516-1530, 2006.

SHELDON, I.M.; CRONIN, J.; GOETZE, L.; DONOFRIO, G.; JOACHIMSCHUBERTH, $\mathrm{H}$. Defining postpartum uterine disease and the mechanisms of infection and immunity in the female reproductive tract in cattle. Biology of Reproduction, v.81, p.1025-1032, 2009.

VERGARA, C.F.; DÖPFER, D.; COOK, N.B.; NORDLUND, K.V.; MCART, J.A.A.; NYDAM, D.V.; OETZEL, G.R. Risk factors for postpartum problems in dairy cows: Explanatory and predictive modeling. J. Dairy Sci., v.97, p.4127-4140, 2014.

VIEIRA, R.J. Obstetrícia em bovinos: da concepção ao puerpério. Acta Veterinária Brasílica, v.8, p.361-368, 2014.

WILLIAMS, E.J. Drivers of post-partum uterine disease in dairy cattle. Reprod. Dom. Anim., v.48, p.53-58, 2013. 